\title{
Modified Foxp3 mRNA protects against asthma through an IL-10-dependent mechanism
}

\author{
Lauren E. Mays, ${ }^{1}$ Susanne Ammon-Treiber, ${ }^{2,3}$ Benedikt Mothes, ${ }^{3}$ Mohammed Alkhaled, ${ }^{1}$ \\ Jennifer Rottenberger, ${ }^{1}$ Eva Sophie Müller-Hermelink, ${ }^{4}$ Melanie Grimm, ${ }^{5}$ \\ Markus Mezger, ${ }^{1}$ Sandra Beer-Hammer, ${ }^{3}$ Esther von Stebut, ${ }^{6}$ Nikolaus Rieber, ${ }^{1}$ \\ Bernd Nürnberg, ${ }^{3}$ Matthias Schwab, ${ }^{2,3}$ Rupert Handgretinger, ${ }^{1}$ \\ Marco Idzko, ${ }^{5}$ Dominik Hartl, ${ }^{1}$ and Michael S.D. Kormann ${ }^{1}$
}

${ }^{1}$ Department of Pediatrics I — Pediatric Infectiology and Immunology, Translational Genomics and Gene Therapy, University of Tübingen, Tübingen, Germany. ${ }^{2}$ Dr. Margarete Fischer-Bosch Institute of Clinical Pharmacology, Stuttgart, Germany. ${ }^{3}$ Institute of Experimental and Clinical Pharmacology and Toxicology, and ${ }^{4}$ Department of Dermatology, University of Tübingen, Tübingen, Germany. ${ }^{5}$ Department of Pneumology, University Hospital Freiburg, Freiburg, Germany. ${ }^{6}$ Department of Dermatology, University Medical Center, Johannes Gutenberg University Mainz, Mainz, Germany.

\begin{abstract}
Chemically modified mRNA is capable of inducing therapeutic levels of protein expression while circumventing the threat of genomic integration often associated with viral vectors. We utilized this novel therapeutic tool to express the regulatory $T$ cell transcription factor, FOXP3, in a time- and site-specific fashion in murine lung, in order to prevent allergic asthma in vivo. We show that modified Foxp 3 mRNA rebalanced pulmonary $T$ helper cell responses and protected from allergen-induced tissue inflammation, airway hyperresponsiveness, and goblet cell metaplasia in 2 asthma models. This protection was conferred following delivery of modified mRNA either before or after the onset of allergen challenge, demonstrating its potential as both a preventive and a therapeutic agent. Mechanistically, FOXP3 induction controlled Th2 and Th17 inflammation by regulating innate immune cell recruitment through an IL-10-dependent pathway. The protective effects of FOXP3 could be reversed by depletion of IL-10 or administration of recombinant IL-17A or IL-23. Delivery of Foxp 3 mRNA to sites of inflammation may offer a novel, safe therapeutic tool for the treatment of allergic asthma and other diseases driven by an imbalance in helper $\mathrm{T}$ cell responses.
\end{abstract}

\section{Introduction}

Allergic asthma is driven by an imbalance in $T$ helper cell responses, leading to chronic airway inflammation, eosinophilia, and mucus hypersecretion in the lung (1). Overactive Th2 responses drive the development of asthma through the production of cytokines and chemokines, which set off a cascade of immune-activating events that lead to airway smooth muscle dysfunction (2). Recent evidence suggests that Th17 cells play a key role in orchestrating allergic airway inflammation by enhancing neutrophil recruitment to the lung $(3,4)$. IL-17 levels are increased in the circulation and airway fluids of asthmatic patients, where they correlate with disease severity (5). Experimental studies report that IL-17 and its upstream mediator, IL-23, can exacerbate or lessen Th2-mediated inflammation in a time- and source-dependent manner (6-9).

Evidence indicates that Tregs, characterized by their transcription factor FOXP3 (10), are capable of suppressing overactive Th2 responses, a mechanism which is likely defective or overridden in patients with allergic disease (11). Airway Tregs are decreased in patients with allergic asthma, showing a reduced suppression of effector $\mathrm{T}$ cell proliferation and cytokine production compared with Tregs in healthy controls (12). Successful corticosteroid treatment is associated with increased Foxp $3^{+}$Treg expression in asthmatic patients and an increased suppressive ability of these cells ex vivo $(12,13)$. Depletion and adoptive transfer studies in animal models have confirmed the ability of Tregs to reduce airway inflammation in vivo $(14,15)$. In fact, experimental exposure to several environmental microbes naturally protects against airway

Conflict of interest: Michael S.D. Kormann is an inventor on a patent licensed to the biopharmaceutical company, Ethris $\mathrm{GmbH}$.

Citation for this article: J Clin Invest. 2013;123(3):1216-1228. doi:10.1172/JCI65351. inflammation by inducing Foxp $3^{+}$Tregs $(16,17)$. Modulation of IL-6, prostanoid, or TNF pathways to enhance the development or recruitment of Tregs has shown similar protective effects (18-20). However, it remains to be seen whether these findings can be successfully translated into a therapeutic agent that enhances Treg function in clinical asthma. Since overexpression of Tregs can also suppress antitumoral or anti-infective immune responses $(21,22)$, the safety of such a treatment would require the induction of Tregs in a controllable and site-specific manner.

We have recently shown life-saving therapeutic efficacy of chemically modified mRNA as a vehicle for upregulating therapeutic levels of protein expression in a rare genetic disease model of surfactant protein B (SP-B) deficiency in the lung (23). Unlike traditional viral vectors, mRNA avoids genotoxicity, as it cannot integrate into the genome. Additionally, mRNA generates naturally self-limited expression, rendering it the ideal vector for controllable induction of Tregs in the airways through mRNA-based upregulation of Foxp3 in the lung. In comparison with recent attempts at systemic Foxp3 upregulation (20), we have optimized a site-specific, highpressure intratracheal spray delivery of modified Foxp 3 mRNA to the lung that is capable of protecting against airway hyperresponsiveness (AHR) and airway inflammation in vivo by modulating both Th2 and Th17 responses in an IL-10-dependent fashion. Delivery of modified Foxp3 mRNA to sites of inflammation may offer a novel, safe, therapeutic tool for the treatment of asthma and other Th2- and IL-17/IL-23-driven diseases.

\section{Results}

Chemically modified mRNA mediates controlled, lung-specific Foxp3 expression. To minimize unwanted off-target effects of Foxp3 upregulation, we aimed to optimize vector formulation and 
A
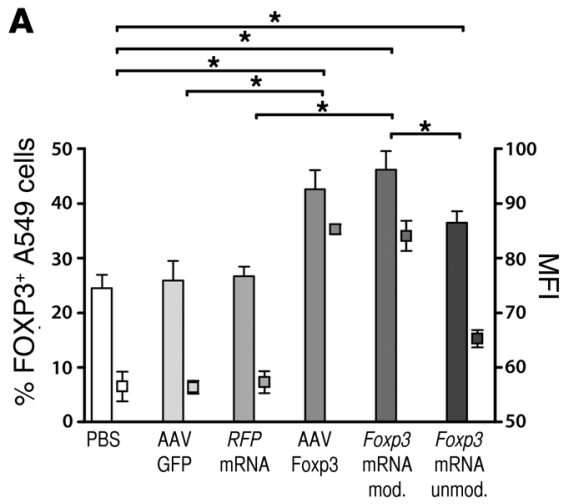

D

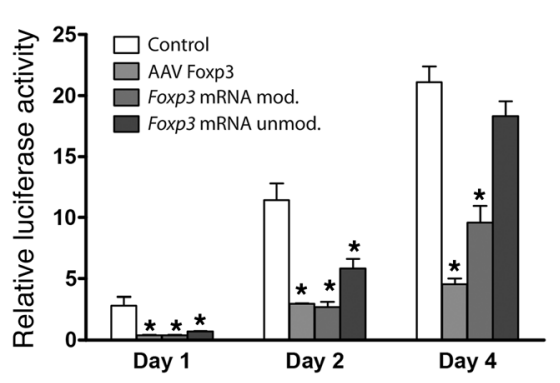

B

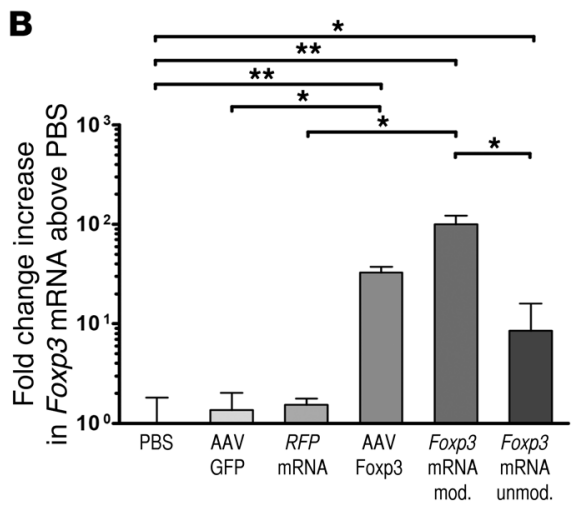

E

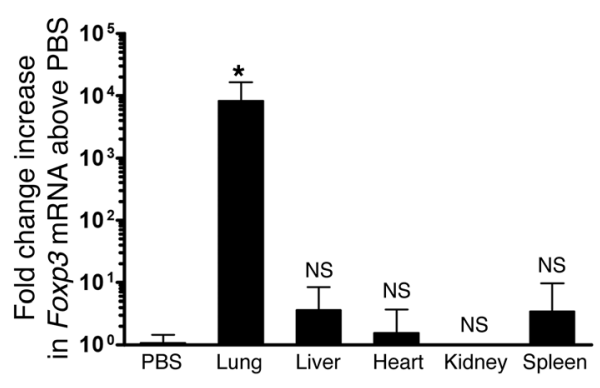

Foxp3 mRNA Foxp3 mRNA

G

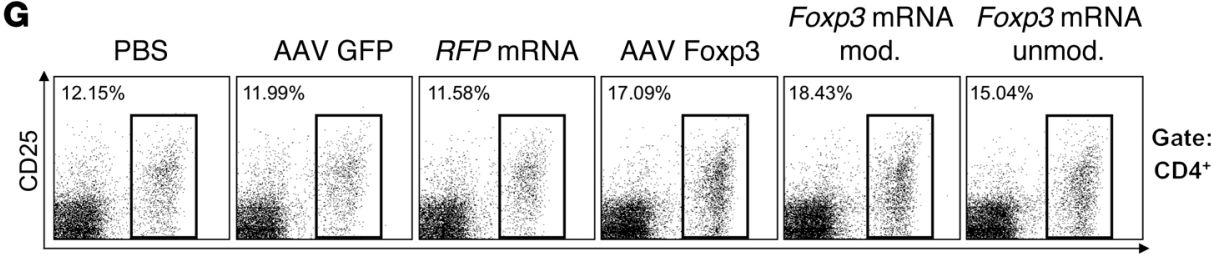

FOXP3

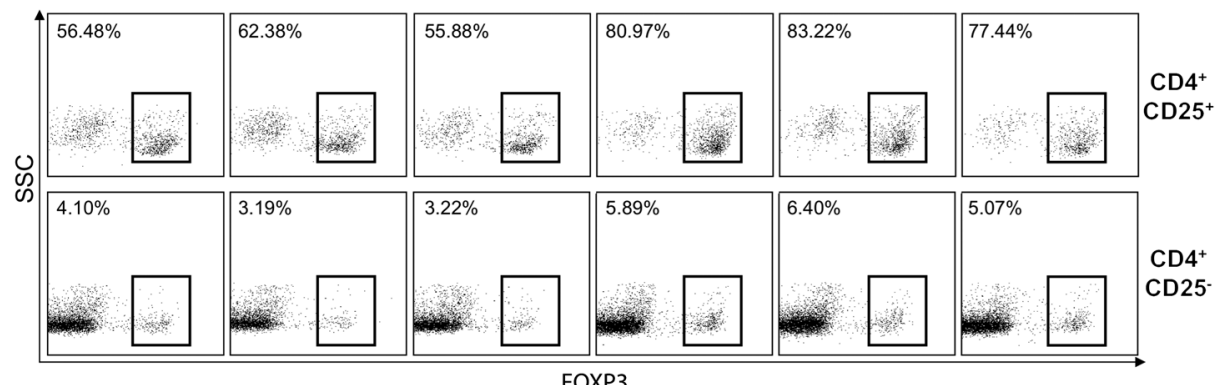

C
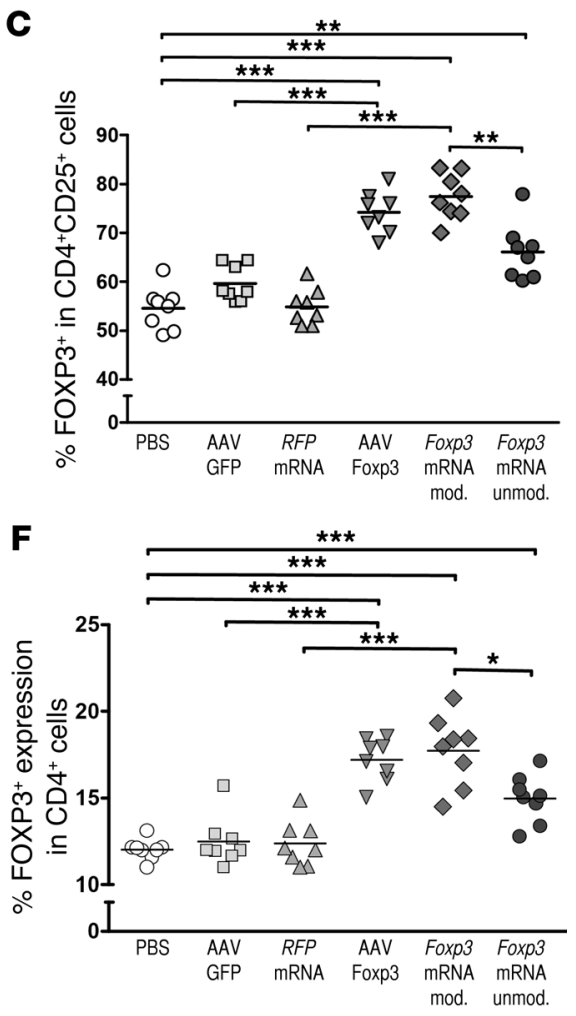

H

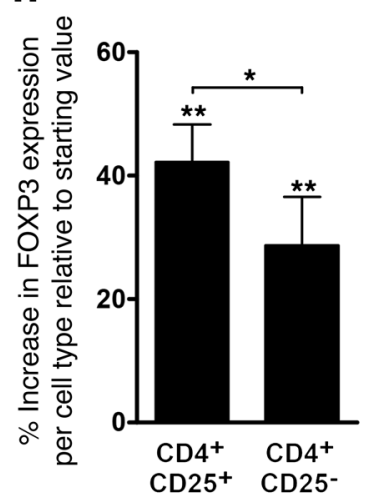

Figure 1

Modified mRNA generates controlled, site-specific expression of Foxp3. Modified Foxp3 mRNA was delivered to A549 cells or was delivered intratracheally, versus unmodified Foxp3 mRNA, modified RFP mRNA, AAV2/6.2.GFP, AAV2/6.2.FOXP3, or PBS. Twenty-four hours after administration, cells or mice were harvested for analysis. (A) In vitro transfection efficiency as reported by the percentage of FOXP3 $3^{+}$A549 cells (left $y$-axis) or MFI (right $y$-axis) by flow cytometry. (B) Cells cotransfected with the indicated vector or control, pNFkB-luc, and an internal Renilla reference plasmid were harvested at the indicated time points and analyzed for luciferase activity. (C) RT-PCR analysis of Foxp3 mRNA expression in lung relative to PBS controls, normalized to B-actin. (D) Biodistribution analysis was performed to monitor organ-specific Foxp3 mRNA expression by RT-PCR. (E-H) Flow cytometric analysis quantifying the percentage of FOXP3 expression in CD4 ${ }^{+} \mathrm{CD} 25^{+}(\mathbf{E})$ and total CD4 ${ }^{+}$cells $(\mathbf{F})$. (G) Representative dot plots showing the percentage of FOXP3 expression per cell type. (H) Percent increase in FOXP3 expression per cell type relative to the starting value, calculated as: (\% FOXP3 in a given cell type for Foxp3 mRNA-injected mice - \% FOXP3 in that cell type for PBS-injected mice)/(\% FOXP3 in that cell type for PBS-injected mice) $x 100 .{ }^{*} P \leq 0.05,{ }^{* *} P \leq 0.01$ and ${ }^{* * *} P \leq 0.001$ for the indicated comparisons, or relative to PBS-injected mice. Data are represented as individual mice or means \pm SD. In vitro studies (A and B) were performed in triplicate and repeated in 3 independent experiments. $(\mathbf{C}-\mathbf{H}) n=6$ mice per group. 
A

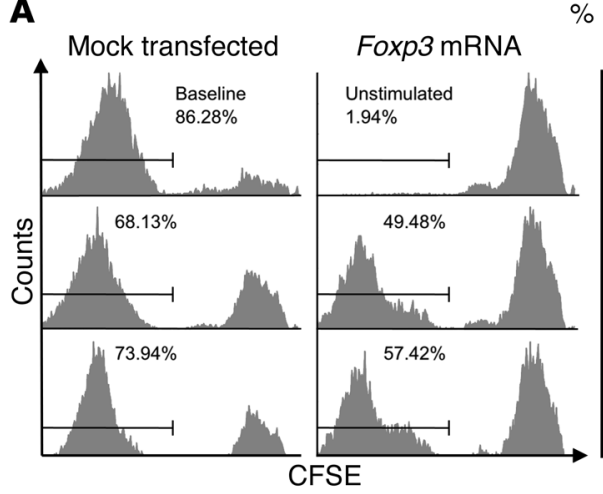

D

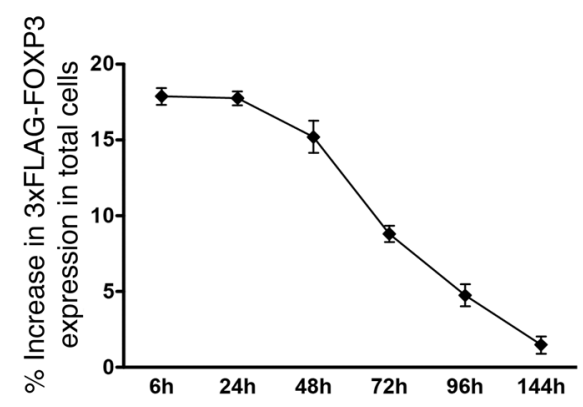

$\%$ Increase in T cell suppression after Foxp3 mRNA

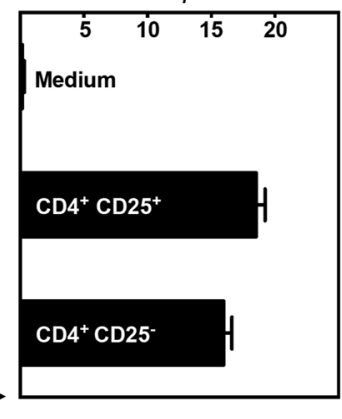

E
B

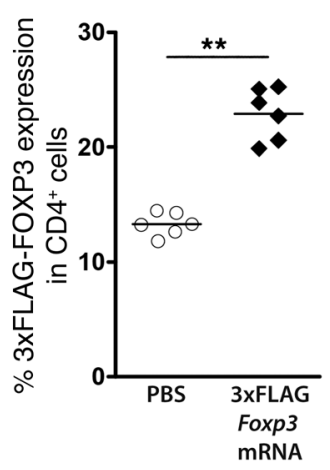

C

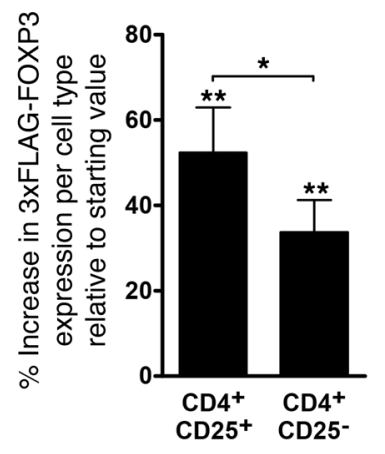

Figure 2

Modified mRNA generates transient upregulation of Foxp3 in CD4+ cells. Modified Foxp3 mRNA (A) or modified Foxp3 mRNA with a 3xFLAG epitope tag (B-E) was delivered to MACS-isolated CD4+ cell populations (A) or intratracheally to mice (B-E), in comparison with PBS. Twenty-four hours after administration, cells or mice were harvested for analysis. (A) Suppression of CFSE-labeled Tresps in vitro, with or without transfection of modified Foxp3 mRNA, and percent increase in suppression following Foxp3 mRNA delivery. Percentage of exogenous 3xFLAGFoxp3 in CD4+ cells (B), and percentage increase of 3xFLAG-Foxp3 in various cell types relative to the starting value (C), or percentage in total cells over time (D) were monitored by flow cytometry. Western blot analysis was performed to detect 3xFLAG-Foxp3 expression in lung (E). (C) Values were calculated as: (\% FOXP3 in a given cell type for Foxp3 mRNA-injected mice - \% FOXP3 in that cell type for PBS-injected mice)/(\% FOXP3 in that cell type for PBS-injected mice) $x 100$. According to this calculation, PBS-injected mice showed a $0 \%$ increase for all cell types. ${ }^{\star} P \leq 0.05,{ }^{* \star} P \leq 0.01$, and ${ }^{* \star *} P \leq 0.001$ for the indicated comparisons between plotted groups, or relative to background levels in PBS-injected mice. Data are represented as individual mice or means \pm SD. In vitro studies in (A) were performed in triplicate and repeated in 3 independent experiments. (B-E) $n=5$ mice per group.

delivery methods that would be capable of achieving efficient yet short-term protein expression in the lung. Therefore, we used a transcriptional therapy approach, where the extent and duration of the encoded protein expression can be precisely controlled, and immune activation largely ablated, by mimicking mammalian mRNA modification schemes during in vitro transcription (23). We transcribed Foxp3 mRNA modified with 10\% 2-thiouridine, 5-methylcytidine (s2UTP/m5CTP) and tested its ability to generate Foxp3 expression in lung cells in vitro (Figure 1A). As expected, modified Foxp3 mRNA significantly increased Foxp3 expression when compared with unmodified transcripts or negative controls, achieving levels comparable to an adeno-associated viral (AAV) vector based on the singleton mutant AAV6.2 (AAV2/6.2.Foxp3), which has been reported to display a high transduction efficiency in murine lung (24) (Figure 1A). Foxp3 expressed by both mRNA and AAV vectors was capable of suppressing NF-kB-dependent luciferase transcription in vitro (Figure 1B). This suppressive effect was increased with the addition of $10 \% \mathrm{~s} 2 \mathrm{UTP} / \mathrm{m} 5 \mathrm{CTP}$ compared with unmodified mRNA, and was functionally comparable to AAV2/6.2 at days 1 and 2 after transfection and transduction. While luciferase remained suppressed by AAV2/6.2.Foxp3 through day 4, expression began to recover in Foxp3 mRNA-transfected cells by day 2 (unmodified) or day 4 (s2UTP/m5CTP-modified) (Figure 1B), confirming reports that nucleoside modifications can increase transcript stability (23). In contrast to viral vectors, which generate longterm protein upregulation and are often associated with risks of genomic integration (25), the shorter half-life and nonintegrating nature of chemically modified mRNA are optimal for achieving fine-tunable Foxp3 expression.

To confirm whether the upregulation of Foxp 3 could also be achieved in vivo, we next injected BALB/c mice with either unmodified or s2UTP/m5CTP-modified Foxp3 mRNA, or AAV2/6.2.Foxp3 as a positive control (Figure 1, C-H). To target Foxp3 overexpression in the lung, we delivered vehicles intratracheally using a high-pressure spraying device, which allows deeper penetration of fine aerosol into the airways (23). RT-PCR and flow cytometric staining demonstrated that Foxp3 mRNA and protein expression levels in the lung following modified mRNA delivery were significantly greater than that from unmodified mRNA and were comparable to AAV2/6.2.Foxp3 (Figure 1, C and E-G). A significant increase in the percentage of Foxp $3^{+}$cells was observed in both the $\mathrm{CD} 4^{+} \mathrm{CD} 25^{+}$Treg fraction, as well as in total $\mathrm{CD}^{+}$cells in comparison with PBS- or 

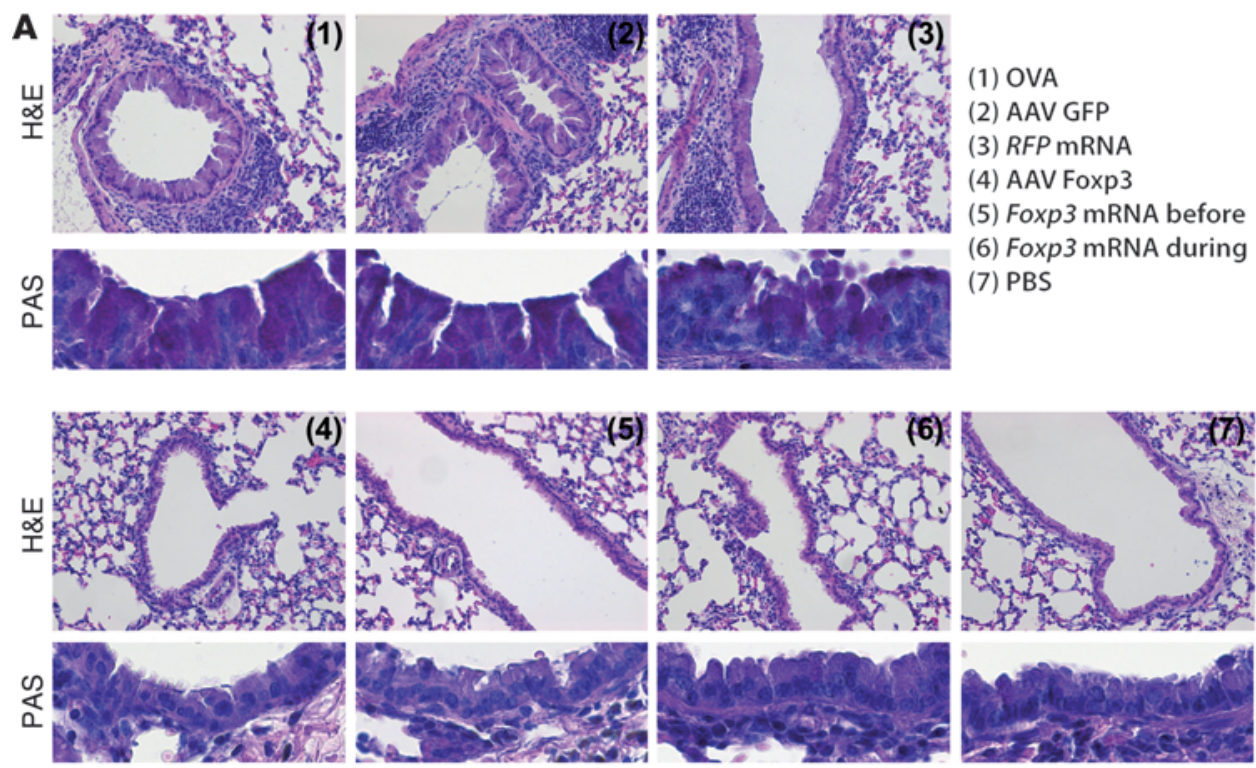

B
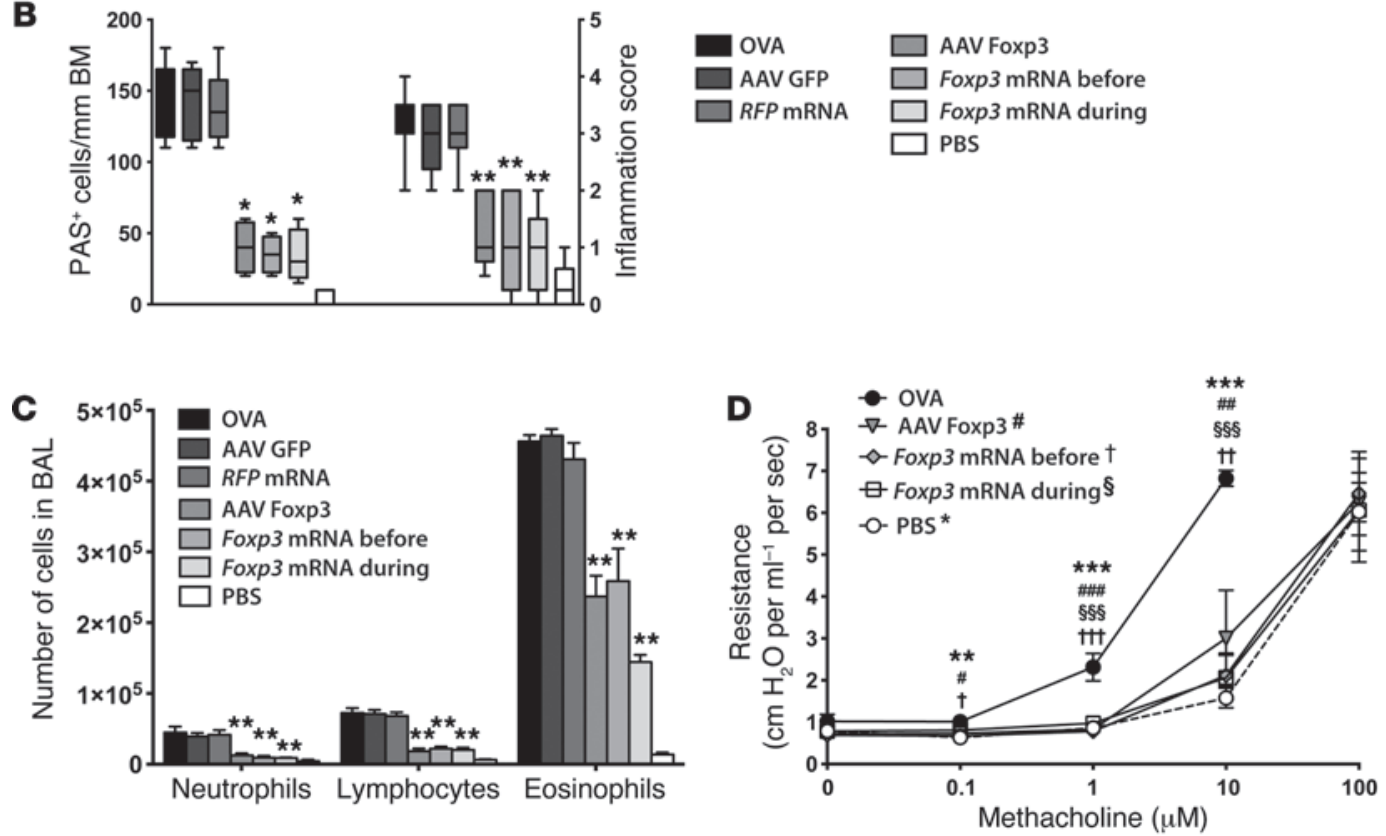

Figure 3

Modified Foxp3 mRNA protects against allergic asthma in mice. Mice sensitized with OVA received modified Foxp3 mRNA either before or during OVA challenge. An AAV2/6.2.Foxp3 vector was used as a positive control. Negative controls included modified RFP mRNA, AAV2/6.2.GFP, and PBS-sensitized/challenged mice. (A and $\mathbf{B}$ ) Tissue inflammation and goblet cell metaplasia analyzed on H\&E- and PAS-stained lung sections. Representative micrographs of H\&E staining (original magnification, $\times 200$ ) and PAS staining (original magnification, $\times 400$ ) are shown in A; scoring for inflammation and PAS ${ }^{+}$cells/mm basement membrane (BM) are shown in B. ${ }^{*} P \leq 0.05$ and ${ }^{* *} P \leq 0.01$ in comparison with OVA-treated, AAV.GFP, or RFP mRNA controls. Data shown as box-and-whisker plots (minimum to maximum, with median line). (C) Differential cell counts after cytospin preparation: the absolute numbers of neutrophils, lymphocytes, or eosinophils in $1 \mathrm{ml}$ of BAL are shown. ${ }^{\star \star} P \leq 0.001$ relative to OVA-treated, AAV. GFP, or RFP mRNA controls. Data are mean \pm SEM. (D) Airway resistance values in response to MCh. ${ }^{*} P \leq 0.05,{ }^{* *} P \leq 0.01$, and ${ }^{* * *} P \leq 0.001$ versus OVA-treated controls. Data are presented as mean \pm SEM. (A-C) $n=8$ mice per group; (D) $n=5$ mice per group.

RFP-GFP vector-injected controls (Figure 1, E-G). To determine whether $\mathrm{CD}^{+} \mathrm{CD} 25^{-}$cells were also capable of taking up Foxp3 vectors, we analyzed the percentage increase in Foxp3 expression per cell type relative to the starting value (Figure $1, G$ and $H$, and Supplemental Figure 1A; supplemental material available online with this article; doi:10.1172/JCI65351DS1). In addition to the significant increase in relative Foxp3 expression in the $\mathrm{CD}_{25^{+}}$Treg cell fraction, $\mathrm{CD} 4^{+} \mathrm{CD} 25^{-}$cells also showed a significant increase in FOXP3 protein expression levels above PBS background (Figure 1H). Furthermore, coculture of lung-iso- 


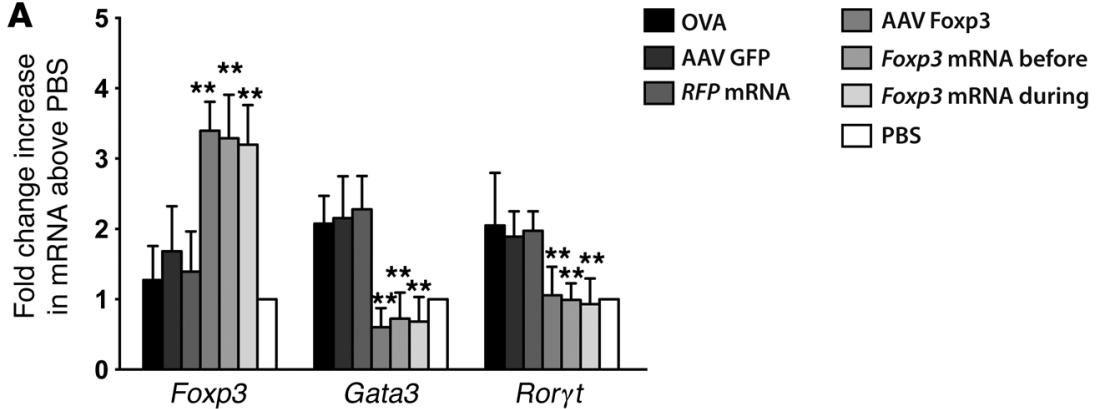

B

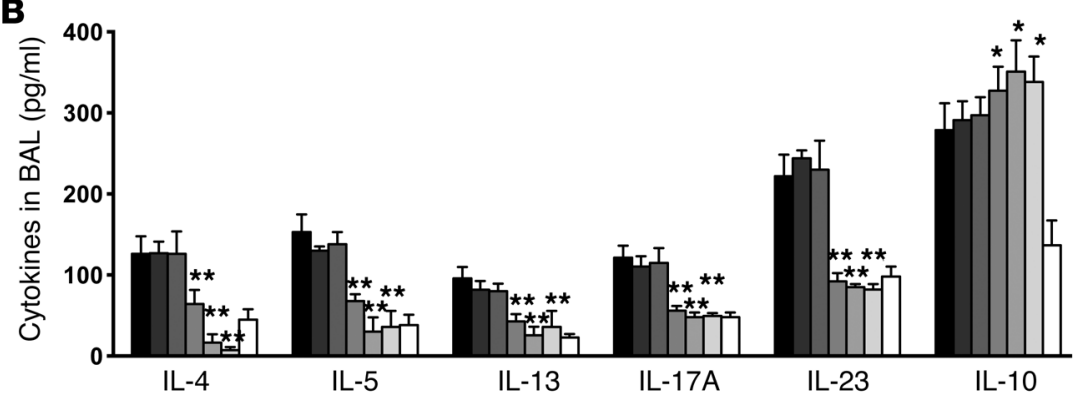

Figure 4

Modified Foxp3 mRNA rebalances $T$ helper responses. Mice sensitized with OVA received modified Foxp3 mRNA either before or during OVA challenge. An AAV2/6.2.Foxp3 vector was used as a positive control. Negative controls included modified RFP mRNA, AAV2/6.2.GFP, and PBS-sensitized/challenged mice. (A) Fold change increase in Foxp3, Gata3, and Roryt mRNA levels relative to PBS controls as detected by RT-PCR and normalized to B-actin. (B) Expression of Th2 cytokines (IL-4, IL-5, and IL-13), Th17 cytokines (IL-17A and IL-23), and IL-10 in BAL by ELISA. (A and B) ${ }^{*} P \leq 0.05$ and ${ }^{* *} P \leq 0.001$ versus OVA-treated, AAV.GFP, or RFP mRNA controls. (C) Levels of OVA-specific IgE in serum by ELISA. ${ }^{*} P \leq 0.05$ and ${ }^{*} P \leq 0.01$ versus OVA- and reporter-treated controls. Data are mean \pm SD. $(\mathbf{A}-\mathbf{C}) n=8$ mice per group.

\section{C}

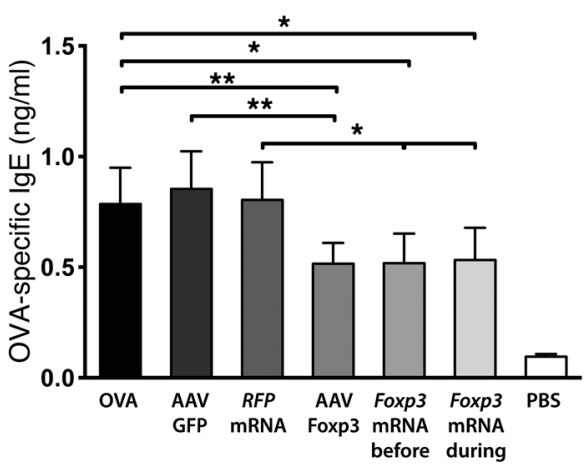

lated $\mathrm{CD} 4{ }^{+} \mathrm{CD} 25^{+}$and $\mathrm{CD} 4{ }^{+} \mathrm{CD} 25^{-}$fractions with CFSE-labeled responder $\mathrm{T}$ cells (Tresps) demonstrated that transfection with Foxp3 mRNA could increase the suppressive potential of both cell populations (Figure 2A). Unexpectedly, an increase in Foxp3 expression was also observed in the $\mathrm{CD}^{-}$- fraction, and transfection of CD4- cells with Foxp3 mRNA ex vivo also resulted in an increased suppressive potential, suggesting that Foxp 3 mRNA may also target $\mathrm{CD}^{+}$or non-T cell populations (Supplemental Figure 1, B and C). To confirm this, modified Foxp3 mRNA containing a $3 x$ FLAG epitope tag was administered intratracheally to discriminate exogenous from endogenous Foxp3 mRNA. Here we observed a similar increase in the percentage of $3 x F L A G-F o x p 3$ expression in $\mathrm{CD}^{+}$cells (Figure $2 \mathrm{~B}$ ), again in both $\mathrm{CD} 4^{+} \mathrm{CD} 25^{+}$and $\mathrm{CD} 4^{+} \mathrm{CD} 25^{-}$cell populations (Figure $2 \mathrm{C}$ ), confirming that the increase observed in Figure 1, E-H, was the result of exogenous mRNA delivery. mRNA-mediated 3xFLAGFOXP3 protein expression was confirmed using flow cytometry and immunoblotting (Figure 2E). Using this FLAG-tagged vector, we also demonstrated that Foxp 3 mRNA could be taken up not only by $\mathrm{CD}^{+}$cell populations, but also by CD4- cells, including airway macrophages, neutrophils, and alveolar type II cells (Figure 2C and Supplemental Figure 1D).
Concomitant with the kinetics of Foxp3-mediated suppression observed in vitro (Figure 1B), we observed a similar time course of 3xFLAG-FOXP3 expression in the lung, where the rapid onset of exogenous mRNA expression occurred as early as 6 hours after delivery, tapering gradually between 48 and 96 hours, and reaching background levels by day 6 (Figure 2D). To address the question of site specificity, a biodistribution analysis was performed, comparing Foxp3 mRNA expression levels in lung, heart, liver, kidney, and spleen 24 hours after injection (Figure 1D). Here, modified Foxp 3 mRNA was delivered 1 and 3 days before sacrifice to mimic the timeline of our asthma model (Supplemental Figure 2). We observed no significant increase in Foxp3 mRNA levels relative to negative controls in any tissue but lung (Figure 1D), confirming that high-pressure intratracheal spray delivery of modified mRNA achieves lung-specific Foxp3 expression.

Lung-specific upregulation of Foxp3 protects against ovalbumin- or house dust mite-induced allergic asthma. In order to test the efficacy of lung-specific Foxp3 upregulation in modulating inflammation, we first performed proof-of-principle studies in an ovalbumin-driven (OVA-driven) mouse model of experimental asthma. Mice received AAV2/6.2-positive control vector 10 days before 
A
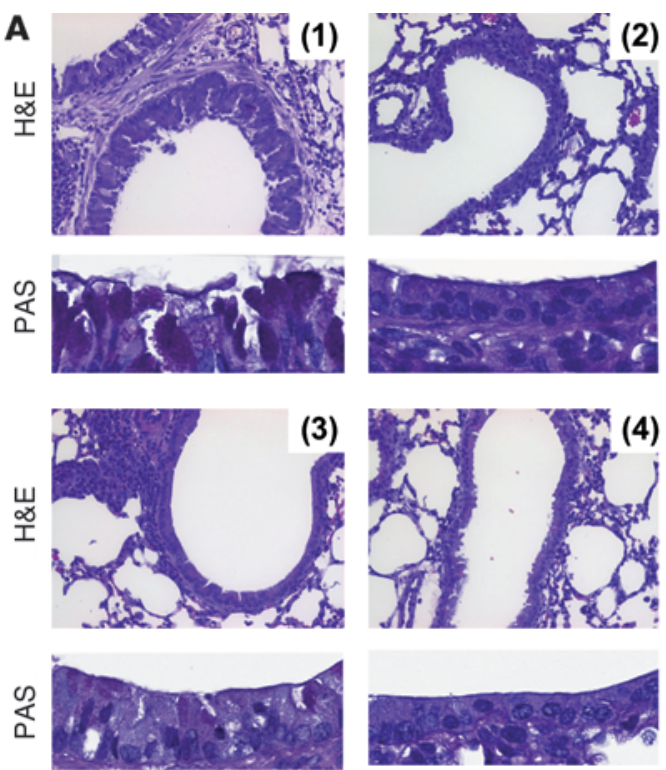

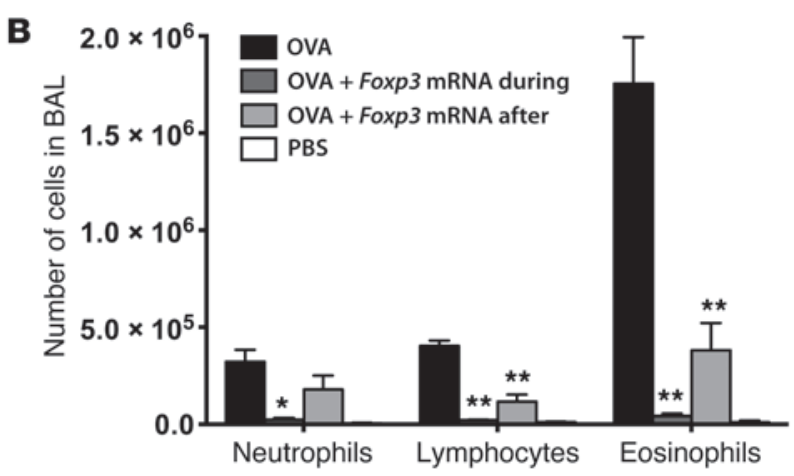

(1) OVA

(2) OVA + Foxp3 mRNA during

(3) OVA + Foxp3 mRNA after

(4) PBS
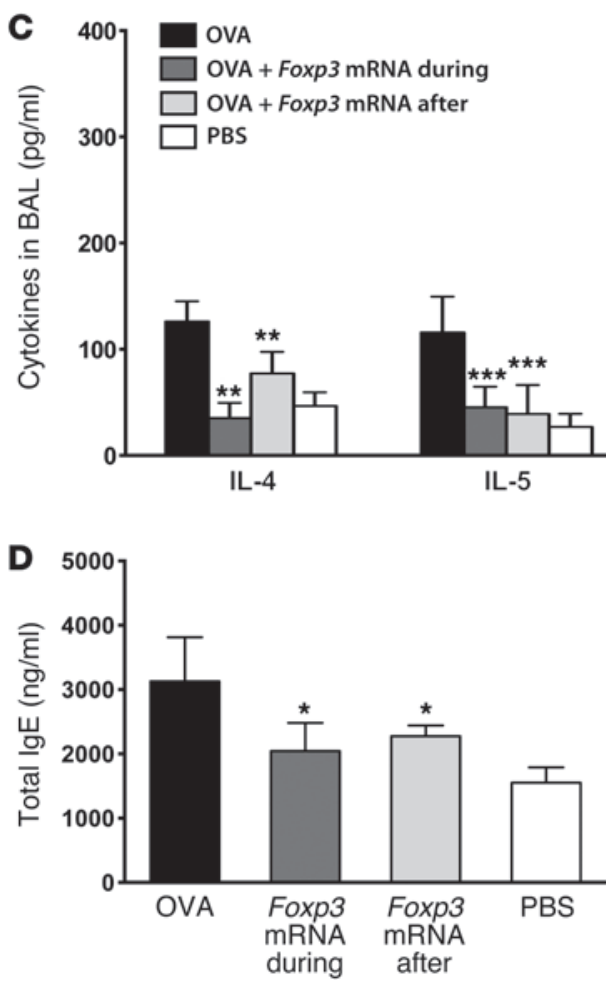

E

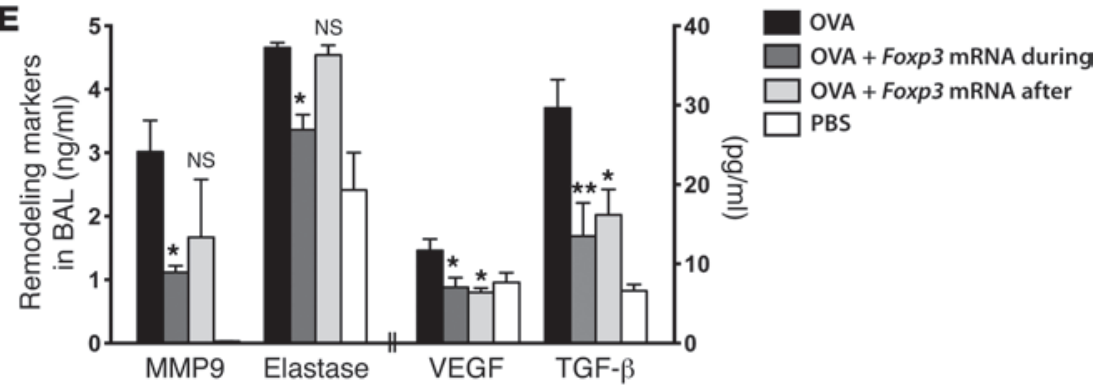

Figure 5

Modified Foxp3 mRNA lowers total IgE and airway remodeling, even when delivered after antigen challenge. OVA-sensitized mice received modified Foxp3 mRNA either during or after the OVA challenge phase. (A) Tissue inflammation and goblet cell metaplasia analyzed on $\mathrm{H} \& \mathrm{E}-$ and PAS-stained lung sections. Representative micrographs of H\&E staining (original magnification, $\times 200)$ and PAS staining (original magnification, $\times 400$ ) are shown. (B) Differential cell counts after cytospin preparation: the absolute number of neutrophils, lymphocytes, or eosinophils in $1 \mathrm{ml}$ of BAL are shown. (C) Expression of Th2 cytokines (IL-4, IL-5, and IL-13), Th17 cytokines (IL-17A and IL-23), and Treg cytokine (IL-10) in BAL by ELISA. Levels of total IgE in serum (D) and airway remodeling markers in BAL (E) by ELISA. (B-E) ${ }^{*} P \leq 0.05$, ${ }^{* *} P \leq 0.01$, and ${ }^{* \star *} P \leq 0.001$ relative to OVA-treated controls. Data are mean $\pm \mathrm{SD}$. (A-E) $n=8$ mice per group. "PBS," "OVA," and "OVA + Foxp3 during" groups were repeated in a total of 3 independent studies. NS, not significant. 
A

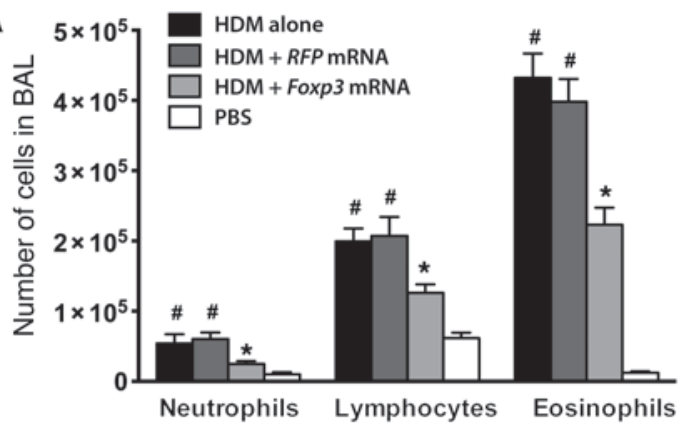

C

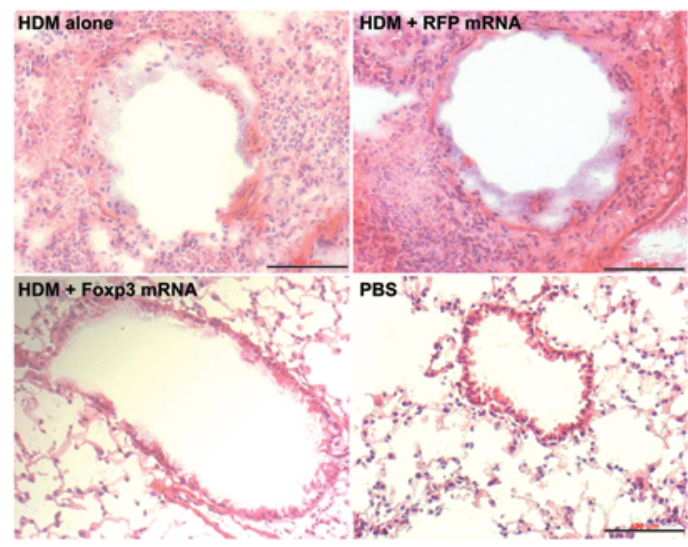

B
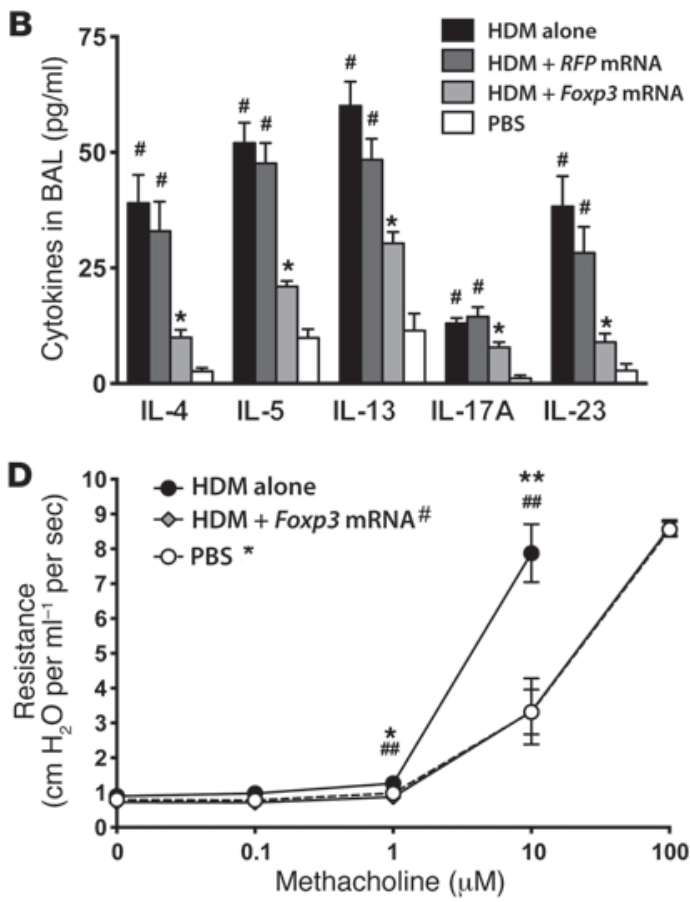

$\mathbf{E}$

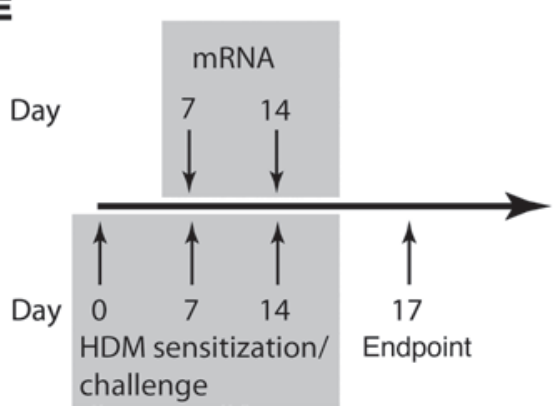

\section{Figure 6}

Modified Foxp3 mRNA demonstrates protection from allergic airway disease in the HDM model. Mice sensitized with HDM received modified Foxp3 mRNA during HDM challenge. Negative controls included modified RFP mRNA and PBS-sensitized mice. (A) Differential cell counts showing the absolute number of neutrophils, lymphocytes, or eosinophils in $1 \mathrm{ml}$ of BAL. (B) ELISAs for expression of IL-4, IL-5, IL-13, IL-17A, and IL-23 in BAL. (A and B) $\# P \leq 0.05$ relative to PBS control, and ${ }^{*} P \leq 0.05$ relative to HDM alone or HDM plus $R F P$ mRNA. (C) Tissue inflammation analyzed on H\&E-stained lung sections. Representative micrographs are shown (original magnification, $\times 200)$. (D) Airway resistance values in response to $\mathrm{MCh}$. ${ }^{\star} P \leq 0.05$ and ${ }^{\star \star} P \leq 0.01$ versus HDM-treated controls. Data are presented as mean \pm SEM. (E) Injection schedule for the HDM-induced model. (A-E) $n=5$ mice per group. Data for $\mathbf{A}-\mathbf{D}$ were acquired in 2 independent studies.

the first aerosol challenge to allow time for transgenic expression (24). Modified Foxp3 mRNA was administered twice, either 1 and 3 days before the first aerosol challenge, or immediately after the first and third challenges, to simulate treatment either before or after the onset of asthma-like symptoms (Supplemental Figure 2). In all cases, Foxp3 delivery to the lung resulted in a significant decrease in peribronchial and perivascular inflammation and goblet cell metaplasia when compared with PBS, RFP mRNA, or AAV2/6.2.GFP negative controls (Figure 3, A and $B$ ). In addition, Foxp3 upregulation led to a significant reduction in BAL eosinophilia, neutrophilia, and lymphocyte infiltration (Figure 3C), while preventing airway hyperresponsiveness, as seen by significantly reduced airway resistance following ex vivo methacholine ( $\mathrm{MCh}$ ) challenge (Figure 3D). Furthermore, ELISAs from BAL supernatant and RT-PCR on
cDNA isolated from lung confirmed the relative increase in Foxp 3 mRNA levels and revealed that the typical upregulation of the Th2 transcription factor, GATA3, and expression of Th2 cytokines, IL-4, IL-5, and IL-13, were significantly corrected following delivery of modified Foxp 3 mRNA (Figure 4, A and B). Elevated levels of OVA-specific IgE observed in serum following antigen exposure were also dampened in the presence of Foxp3 mRNA (Figure 4C). OVA-specific serum IgE, cellular infiltration, and Th2 responses in BAL and lung remained elevated for RFP mRNA and AAV2/6.2.GFP controls compared with PBS-aerosolized control mice.

Notably, protection from airway hyperresponsiveness, Th2 responses, and cellular infiltration was observed in mice receiving modified Foxp 3 mRNA either before or during the aerosolized OVA challenge, suggesting that this method may have 
A
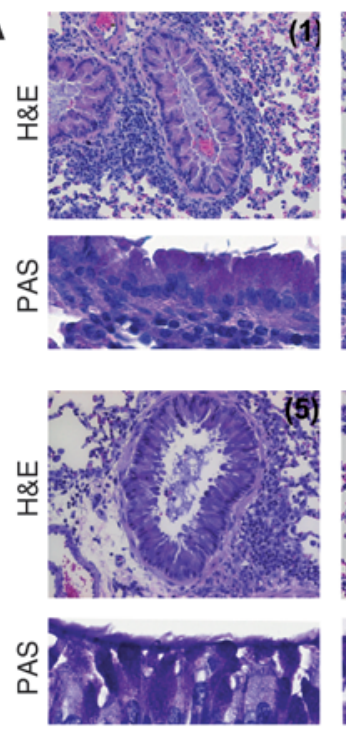

B

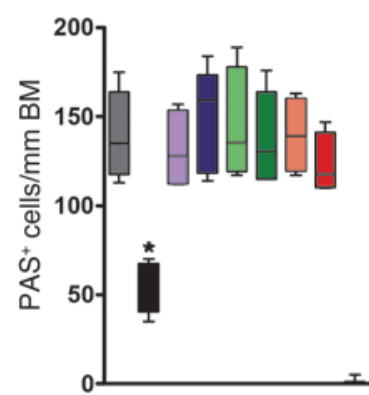

PAS
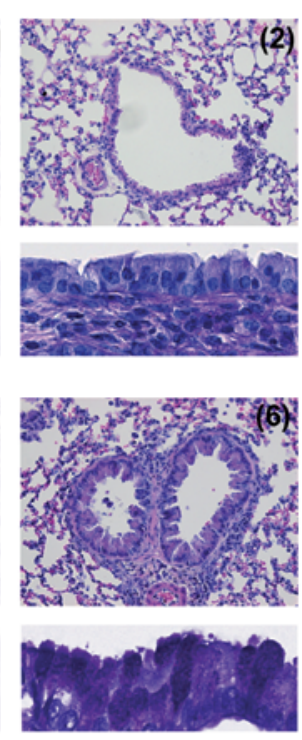
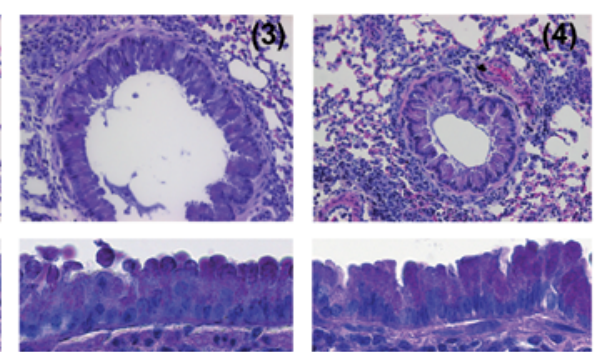

(1) OVA

(2) OVA + Foxp 3

(3) OVA + rIL-17A

(4) OVA + rIL-17A + Foxp3

(5) OVA + rlL-23

(6) OVA + rIL-23 + Foxp3

(7) OVA + all-10

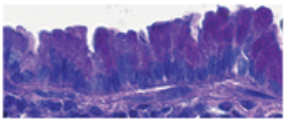

(8) OVA + $\alpha$ IL-10 + Foxp3

(9) PBS
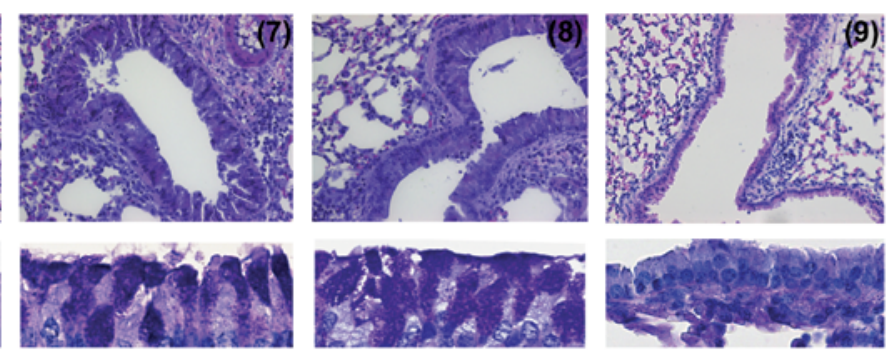

C

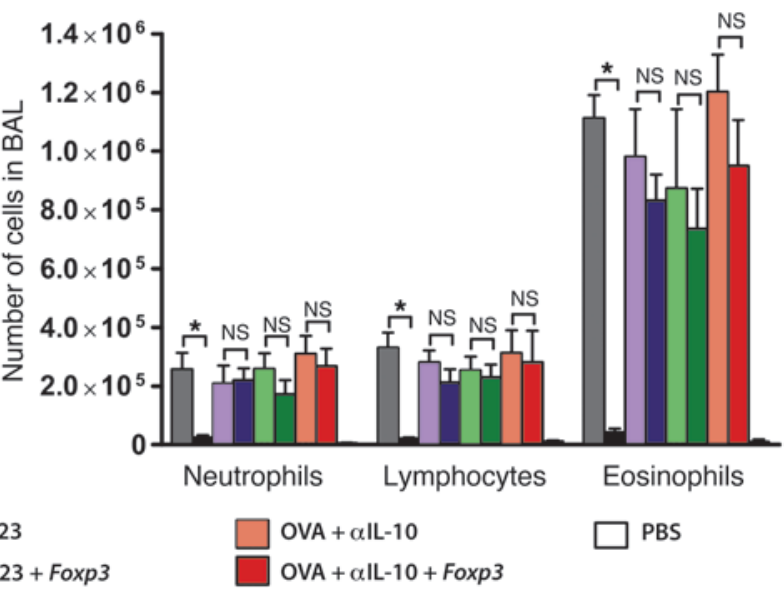

\section{Figure 7}

Recombinant IL-17A/IL-23 or depletion of IL-10 ablates the protective effects of modified Foxp3 mRNA. Mice were sensitized and challenged with OVA or PBS. During challenge, mice received modified Foxp3 mRNA either alone or in combination with recombinant IL-17A or IL-23. Some groups received an IL-10-depleting antibody before the onset of OVA challenge. (A and B) Tissue inflammation and goblet cell metaplasia analyzed on H\&E- and PAS-stained lung sections. Representative sections of H\&E staining (original magnification, $\times 200)$ and PAS staining (original magnification, $\times 400$ ) are shown in $\mathbf{A}$; scoring for inflammation and $\mathrm{PAS}^{+}$cells is shown in $\mathbf{B}$. Data are shown as box-and-whisker plots (minimum to maximum, with median line). (C) Differential cell counts showing absolute numbers of neutrophils, lymphocytes, or eosinophils in $1 \mathrm{ml}$ of BAL. ${ }^{\star} P \leq 0.05 ;{ }^{* *} P \leq 0.01$; ${ }^{* *} P \leq 0.001$ (mean $\pm \mathrm{SD}$ ). (A-C) $n=8$ mice per group. "PBS," "OVA," and "OVA + Foxp3" groups were also repeated in a total of 3 independent studies. r, Recombinant.

both preventive and therapeutic applications (Figures 3 and 4). Promisingly, partial protection from these phenotypes could also be achieved following delivery of modified Foxp3 mRNA even after the final OVA challenge, including a reduction in goblet cell metaplasia, eosinophil and lymphocyte infiltration, as well as IL-4 and IL-5 cytokine levels (Figure 5, A-C). Delivery of Foxp3 mRNA during or after challenge also reduced the levels of total serum IgE and markers of airway remodeling (Figure 5, D and E).

Next, to better mimic the human condition, we investigated the effect of Foxp 3 mRNA in a house dust mite-driven (HDMdriven) model of experimental asthma in which sensitization occurs via the mucosa of the airways. Strikingly, concomitant with our findings in the OVA model, Foxp3 upregulation in the HDM model was associated with a significant reduction in neu- trophil, lymphocyte, and eosinophil numbers in BAL (Figure 6A), diminished BAL levels of Th2 cytokines (Figure 6B), and reduced peribronchial and perivascular tissue infiltration in lung sections (Figure 6C). Airway hyperresponsiveness was also prevented, as determined by significantly lower airway resistance following ex vivo MCh challenge (Figure 6D). In summary, these data show that intratracheal application of modified Foxp 3 mRNA protects against allergic airway disease in 2 well-established mouse models of experimental asthma.

Foxp3-mediated protection from overactive Th2 responses is dependent on control of the Th17 response. Since there is a reported link between Th17 cells and neutrophil infiltration $(3,4)$ and the increase in neutrophils observed in our models of allergen-induced airway inflammation (Figure 3C and Figure 6A), we next 

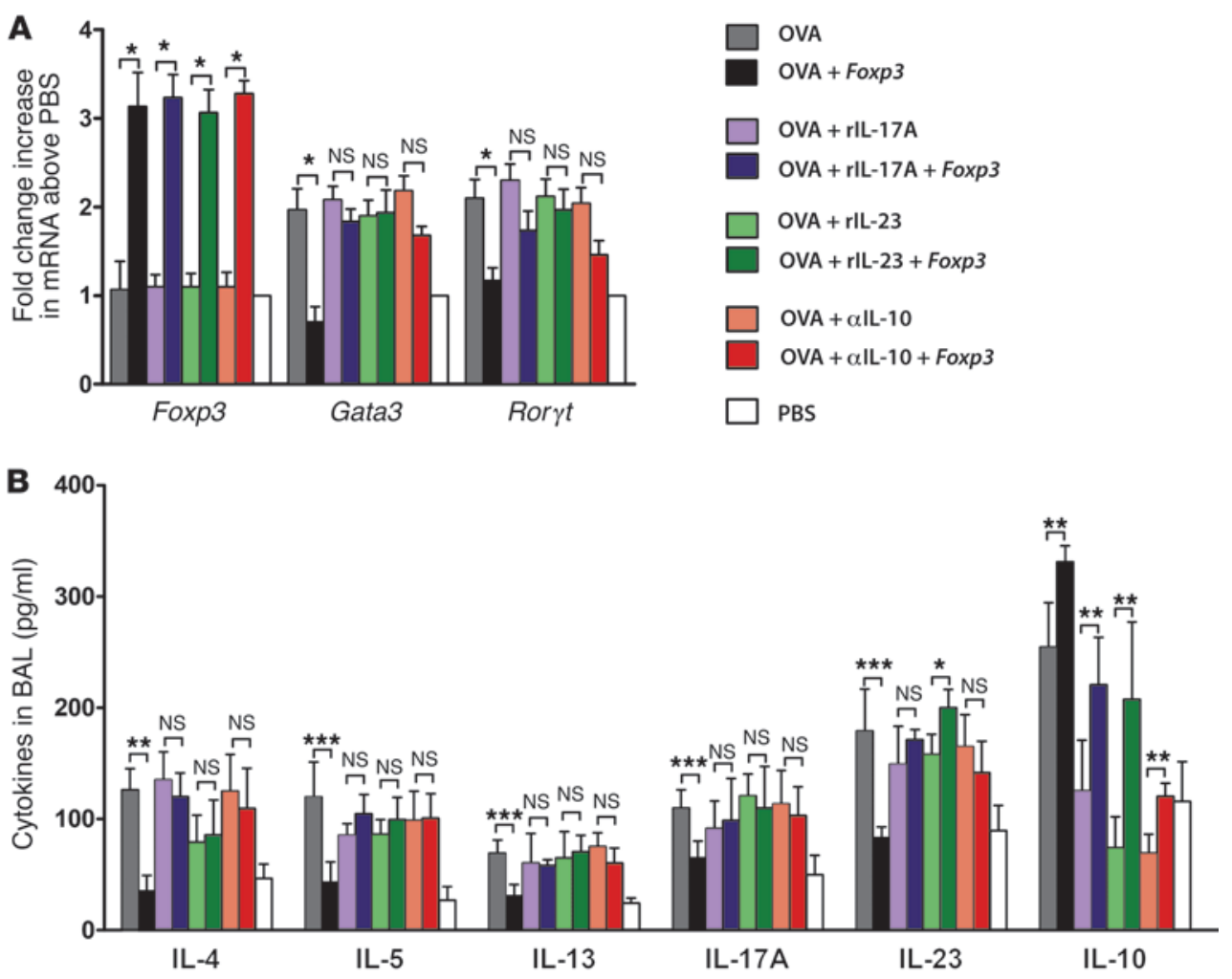

\section{Figure 8}

Helper T cell responses are no longer rebalanced in the presence of recombinant IL-17A/IL-23 or depletion of IL-10. Mice were sensitized and challenged with OVA or PBS. During challenge, mice received modified Foxp3 mRNA either alone or in combination with recombinant IL-17A or IL-23. Some groups received an IL-10-depleting antibody before the onset of OVA challenge. (A) Fold change increase in Foxp3, Gata3, and Roryt mRNA levels relative to PBS controls as detected by RT-PCR and normalized to B-actin. (B) Expression of Th2 cytokines (IL-4, IL-5, and IL-13), Th17 cytokines (IL-17A and IL-23), and Treg cytokine (IL-10) in BAL by ELISA. ${ }^{\star} P \leq 0.05,{ }^{\star \star} P \leq 0.01$, and ${ }^{* * *} P \leq 0.001$ (mean $\pm \mathrm{SD}$ ). (A and B), $n=8$ mice per group. "PBS," "OVA," and "OVA + Foxp3" groups were also repeated in a total of 3 independent studies. investigated the role of Th17 responses in our Foxp 3 mRNA treatment model. Indeed, expression of the Th17 transcription factor ROR $\gamma \mathrm{t}$ in the lung tissue and expression of IL-23 and IL-17A in BAL supernatants were upregulated following allergen sensitization and challenge (Figure 4, A and B, and Figure 6B). ROR $\gamma$ t is reported to specifically determine Th17 lineage development and is mutually exclusive in Foxp3 expression in $\mathrm{CD}^{+} \mathrm{T}$ cells, as Foxp 3 inhibits ROR $\gamma t$ expression and Th17 development (26). IL-23 expression has been reported to play a role in promoting Th17 expansion by directly affecting $T$ cells and initiating the release of TGF- $ß$ and IL- 6 by DCs (8). Notably, in Foxp3 mRNA-treated mice, ROR $\gamma t$, IL-23, and IL-17A expression levels were significantly downregulated, concomitant with the significant improvement observed in AHR, cellular infiltration, and rebalancing of Th2 responses (Figure 3, 4, and 6). Confirming the effect of modified Foxp3 mRNA on Th17 levels, we also observed that the percentage of IL-17A expression in the $\mathrm{CD} 4^{+}$cell population was significantly decreased 24 hours after Foxp3 mRNA delivery to the lung (Supplemental Figure 3).

To corroborate the mechanistic importance of Th17 responses in Foxp3-mediated asthma protection, recombinant IL-17A or IL-23 proteins were administered simultaneously with modified Foxp3 mRNA during aerosolized OVA challenge. It has been previously reported that IL-23 and Th17 cells can exacerbate Th2-driven eosinophilic airway inflammation (7). In the presence of exogenous Th17 stimulation, the protection conferred by modified Foxp3 mRNA was largely abolished, and phenotypes of airway disease returned, such as peribronchial and perivascular infiltration (Figure 7A and B), increased lymphocyte infiltration, eosinophilia and neutrophilia (Figure 7C), and expres- sion of Gata3 in the lung (Figure 8A) and Th2 cytokine levels in BAL (Figure 8B) - all despite the preservation of elevated Foxp3 mRNA expression levels in the lung (Figure 8A).

To further support the role of IL-17A in this model, experiments were repeated in IL-17A-deficient mice. As previously reported, the degree of inflammation, infiltration, and Th2 activation following allergen sensitization and challenge was significantly reduced in IL-17A knock-outs versus wild-type control mice (Figure 9; data not shown) (27). Even in the absence of IL-17A, administration of modified Foxp3 mRNA during challenge still showed a significant decrease in airway inflammation, cellular infiltration, IL-4, -5, -13, and -23 cytokine levels, total serum IgE, and airway remodeling factors (Figure 9).

Foxp3-induced asthma protection is mediated through an IL-10-dependent mechanism. A large body of evidence suggests that Foxp3mediated control of Th2- and/or Th17-related inflammation is associated with an increase in the antiinflammatory cytokine IL-10 (28-32). Interestingly, following delivery of modified Foxp3 mRNA, we observed a significant increase in IL-10 protein levels in the BAL fluid (Figure 4B). To determine if modified Foxp3 mRNA mediates its protective effects through IL-10, we repeated our model, depleting IL-10 throughout the challenge phase. Here we observed that Foxp3-mediated protection was completely reversed in the absence of IL-10, as seen by a return of airway inflammation and goblet cell metaplasia (Figure 7, A and B). Furthermore, the significant reduction in neutrophil, eosinophil, and lymphocyte infiltration, as well as the downregulation of Th2 and Th17 transcription factors and cytokine levels after Foxp3 mRNA treatment, were not observed in mice depleted of IL-10 (Figure 7C and Figure 8). 
A
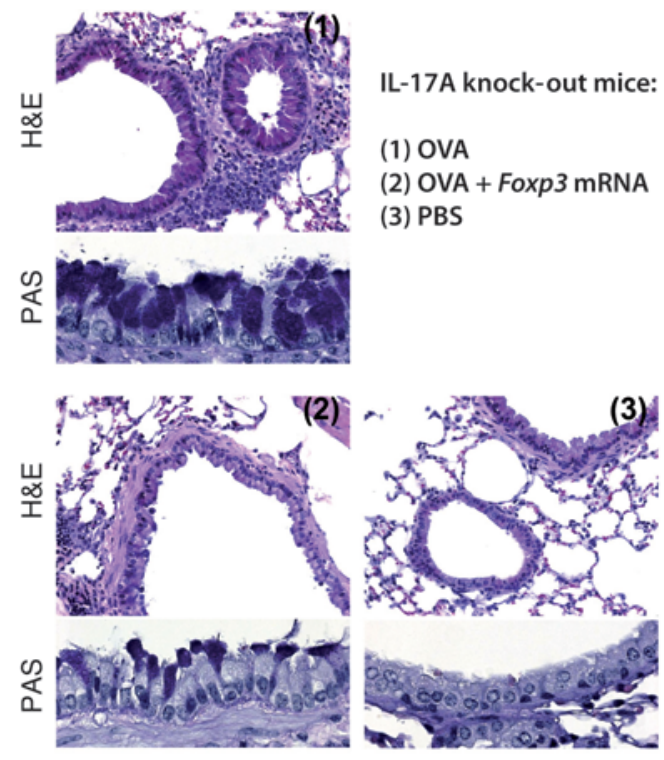

\section{B}

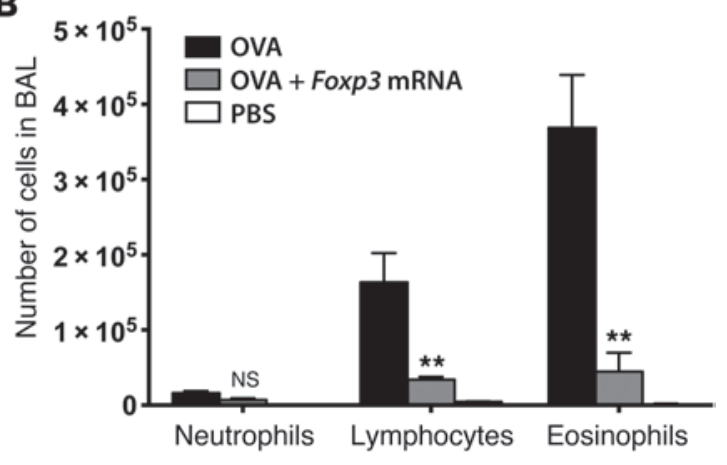

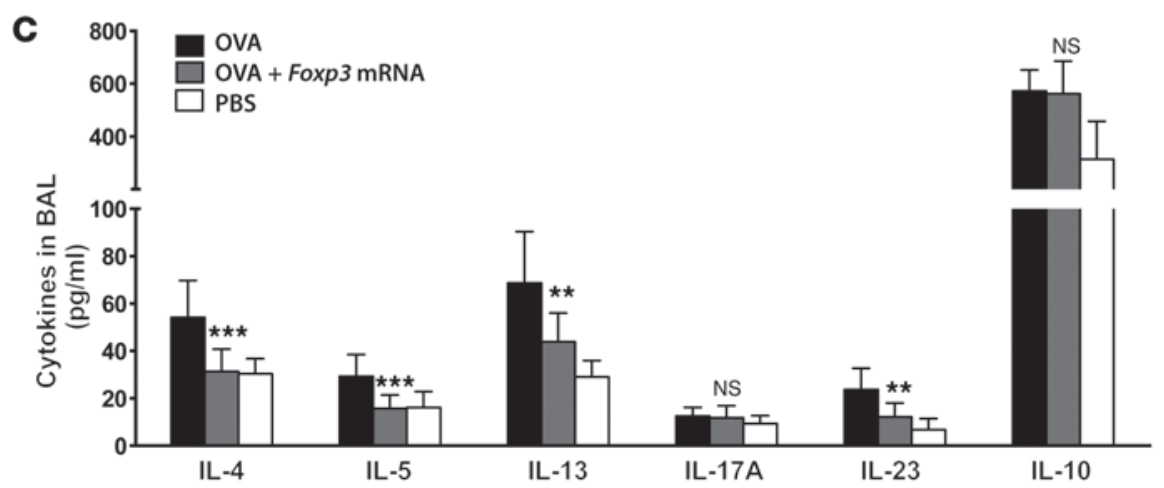
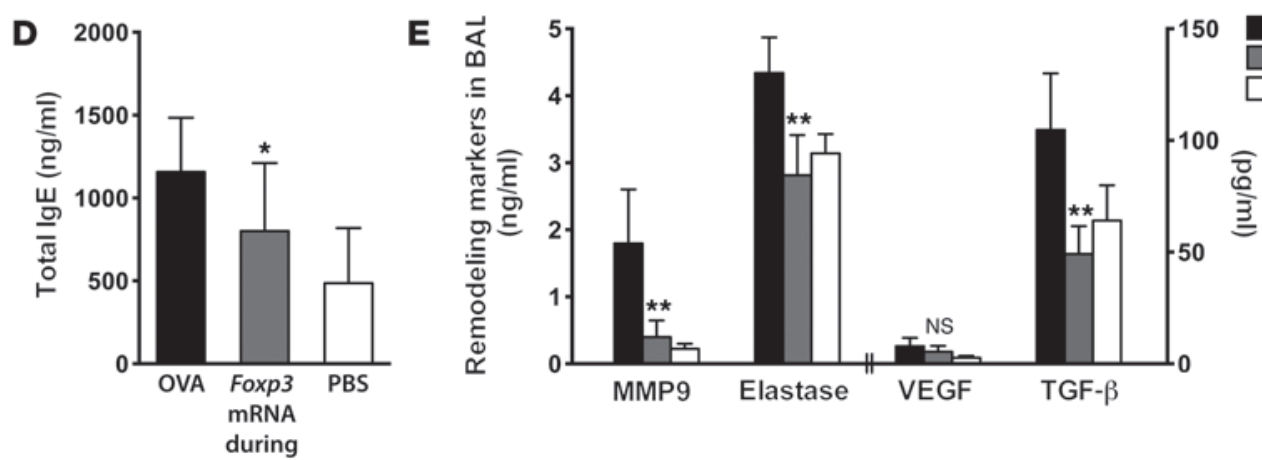

\section{Figure 9}

Effects of modified Foxp3 mRNA in IL-17A knock-out mice. IL-17A ${ }^{-/-}$mice were sensitized and challenged with OVA in the presence or absence of modified Foxp3 mRNA during the challenge phase ( $n=5$ mice per group). (A) Tissue inflammation and goblet cell metaplasia analyzed on H\&E- and PAS-stained lung sections. Representative micrographs of H\&E staining (original magnification, $\times 200$ ) and PAS staining (original magnification, $\times 400$ ) are shown. (B) Differential cell counts after cytospin preparation: the absolute numbers of neutrophils, lymphocytes, or eosinophils in $1 \mathrm{ml}$ of BAL are shown. (C) Expression of Th2 cytokines (IL-4, IL-5, and IL-13), Th17 cytokines (IL-17A and IL-23), and IL-10 in BAL by ELISA. Levels of total IgE in serum (D) and airway remodeling markers in BAL (E) by ELISA. (B-E) ${ }^{*} P \leq 0.05$, ${ }^{* *} P \leq 0.01$, and ${ }^{* * *} P \leq 0.001$ for OVA + Foxp3 relative to OVA-treated controls. Data are mean \pm SD.

\section{Discussion}

In a setting where the rebalancing of helper $\mathrm{T}$ cell responses is desired, upregulation of the regulatory transcription factor FOXP3 holds great therapeutic potential. Importantly, however, the ability to induce Foxp3 in a controllable, short-term, and site-specific fashion is critical to the development of safe therapeutics, as Tregs are also capable of suppressing antitumoral and anti-infective immune responses, and overexpression of suppressive cells has been associated with the progression and metastasis of some cancers and infectious diseases $(21,22)$. In contrast 
to viral vectors that generate long-term protein upregulation and are often associated with risks of genomic integration (25), the shorter half-life and nonintegrating nature of chemically modified mRNA render it optimal for achieving fine-tunable Foxp3 expression. It remained to be seen, however, whether this novel vector could deliver levels of in vivo protein expression comparable to those of efficient viral vectors, such as AAV. This report is the first known direct comparison of modified mRNA transcripts with a well-characterized AAV vector, showing that it is possible for modified mRNA to obtain equivalent therapeutic levels of protein expression in murine lung compared with a low-dose, lung-efficient AAV2/6.2 vector. This finding is important because it confirms that modified mRNA is a safe and competitive therapeutic tool for applications where efficient, nonintegrating, controlled, and repeatable short-term expression are desired. By following this strategy, we have optimized an ideal vehicle and a delivery method for achieving an efficient, yet transient, site-specific increase in Foxp3 expression in murine lung using a vector that avoids genomic integration and is amenable to repeated administration.

Furthermore, through intratracheal application of modified Foxp3 mRNA to the lung, we were able to achieve protection against allergic airway disease phenotypes in 2 well-established mouse models of experimental asthma. This was achieved by rebalancing pulmonary Th2 responses, reducing cellular eosinophilic and neutrophilic infiltration and peribronchial inflammation, and by improving lung function in response to $\mathrm{MCh}$. Promisingly, these protective effects were observed not only when modified mRNA was delivered before the onset of antigen challenge, but also when delivered during or even after the antigen challenge phase. These findings suggest that modified Foxp 3 mRNA may have both preventive and therapeutic applications, with implications for additional translational studies aiming beyond allergic asthma.

Mechanistically, these studies showed that modified Foxp3 mRNA delivery was responsible for rebalancing not only Th2 responses, but also overactive Th17 responses. A similar role for Tregs in controlling Th17 cells in a mouse model of inflammatory colitis has recently been reported (27). When looking more closely at the role of Th17 in this setting, we observed that exogenous IL-17A or IL-23 proteins could reverse the protection from asthmatic disease conferred by Foxp3, providing evidence that modified Foxp3 mRNA functions by rebalancing both Th2 and Th17 responses. Control of Th17 responses appeared critical to the improvement of the asthmatic phenotype in this system, tempting us to speculate that the therapeutic efficacy of Foxp3 may be compromised in a setting of excessive Th17 levels. In matched knock-out mice with an absence of IL-17A, the asthmatic phenotype was also reduced, and administration of modified Foxp3 mRNA during challenge showed further, significant phenotypic improvement. When viewed in combination, these findings support the notion that the Treg-Th17 balance may dictate whether an inflammatory response can be activated or controlled. We hypothesize that Th2 dysregulation and asthma development in this system is driven by the intimate balance between Treg and Th17 responses, acting in dissonance to either suppress or activate an inflammatory environment. Transcript-based upregulation of Foxp3 expression was capable of tipping the balance toward suppression and control of Th2-driven airway inflammation, a phenotype that was further supported by the absence of IL-17A in knock-out mice, or was counteracted and ablated upon exogenous delivery of recombinant IL-17A or IL-23. However, whether a causal link between Th2 and Th17 immune responses exists in this system remains a topic for further investigation.
It was recently discovered that Th17 cells express the receptor for IL-10 and are thus controlled by Foxp $3^{+}$Tregs in an IL-10-dependent manner (27). In the present study, we also observed an IL-10 dependency of Foxp3-mediated protection, where the asthmatic phenotype returned upon depletion of IL-10. In combination, these findings support our conclusion that Foxp3 mRNA-mediated protection against Th2- and Th17-derived airway inflammation functions through an IL-10-dependent mechanism.

In conclusion, we have demonstrated a novel, IL-10-mediated, therapeutic approach to treating allergic asthma with the use of modified Foxp3 mRNA. We have optimized a high-pressure intratracheal spray delivery of modified mRNA to the lung and observed that, unlike stably expressing vectors or systemic delivery methods, we achieved efficient, repeatable, site-specific, and naturally self-limiting upregulation of FOXP3 protein expression in the lung. The addition of double modifications allowed mRNA to achieve expression levels comparable to those in an $\mathrm{AAV}$ vector known for its high transduction efficiency in murine lung. Foxp3 upregulation conferred protection from AHR, lung inflammation, eosinophilia, and overactive Th2 responses in 2 mouse models of allergic asthma. In parallel, modified Foxp 3 mRNA treatment was also effective in downregulating allergen-induced neutrophilia and overactive Th17 responses, and its protective effects could be reversed by depletion of IL-10 or administration of recombinant IL-17A or IL-23. When viewed in combination, these findings suggest that maintaining a balance between Treg, Th2, and Th17 responses may be critical in controlling the asthmatic response, and that IL-10 plays a key role in the Treg-mediated effects in this scenario. Since delivery of Foxp3 mRNA was able to protect against airway disease before or after the onset of allergen challenge, modified Foxp3 mRNA represents an ideal tool for both preventive and therapeutic strategies in allergic asthma and beyond.

\section{Methods}

\section{$m R N A$ and $A A V$ vector production}

mRNA transcripts were produced as previously described (23). Briefly, a T7 promoter-containing pVAX1 plasmid (Life Technologies) with a 120 bp polyA tail expressing mouse Foxp3 cDNA (CCDS 29965.1) was linearized by $\mathrm{XbaI}$ and transcribed in vitro using the mMESSAGE mMACHINE T7 ULTRA kit (Life Technologies), incorporating 10\% 2-Thio-UTP and 5-Methyl-CTP (TriLink BioTechnologies). Recombinant AAV2/6.2 vectors expressing either EGFP or Foxp3 were created, as previously described, using plasmids obtained from the Penn Vector Core (24). Vectors were purified by iodixanol gradient centrifugation (Sigma-Aldrich) and an Amicon-Ultra Centrifugal Filter Unit (EMD Millipore) for buffer exchange and concentration.

\section{In vitro experiments}

Human alveolar type II epithelial cells (A549) were grown in Dulbecco's Minimum Essential Media (Life Technologies), supplemented with 10\% FCS and $1 \%$ penicillin-streptomycin. Twenty-four-well plates seeded with 80,000 cells per well were transfected with mRNA (200 ng), AAV (MOI 10 $)$, and/or $1 \mu \mathrm{g}$ pNFkB-luc (Clontech) using Lipofectamine 2000 (Life Technologies) according to the manufacturer's instructions. Luciferase activity was analyzed using the Ready-To-Glow Dual Secreted Reporter Assay (Clontech).

\section{CFSE suppression assays}

$\mathrm{CD}^{-}, \mathrm{CD}^{+} \mathrm{CD} 25^{-}$, and $\mathrm{CD}^{+} \mathrm{CD} 25^{+}$fractions were isolated from $\mathrm{BALB} / \mathrm{c}$ spleen using magnetic activated cell-sorting beads (MACS; Miltenyi Biotec). $\mathrm{CD}^{+}{ }^{+} \mathrm{CD} 25^{-}$Tresps were labeled with CFSE (CellTrace; Life Technologies) 
and cocultured 1:1 with non-labeled $\mathrm{CD} 4^{-}, \mathrm{CD} 4^{+} \mathrm{CD} 25^{-}$, or $\mathrm{CD} 4^{+} \mathrm{CD} 25^{+}$ cells that had been transfected with either PBS (mock) or $500 \mathrm{ng}$ modified Foxp 3 mRNA. Cells were cultured for 3 days in the presence of CD3 and CD28 (T Cell Activation/Expansion Kit, mouse; Miltenyi Biotec), and analyzed for CFSE dilution by flow cytometry.

\section{Animal experiments}

Six- to eight-week-old female BALB/c mice were purchased from Charles River Laboratories. Animal procedures were approved by the local ethics committee and carried out according to the guidelines of the German Law for the Protection of Animals. Intratracheal and intranasal procedures were carried out under antagonizable anesthesia. Asthma models are summarized in Supplemental Figure 1. Briefly, mice were sensitized with $50 \mu \mathrm{g}$ OVA (Sigma-Aldrich) s.c. and challenged with 1\% aerosolized OVA for 30 minutes using an ultrasonic nebulizer (PARI BOY SX; PARI GmbH). HDM extracts $(100 \mu \mathrm{g})$ were delivered intratracheally. AAV vectors $\left(5 \times 10^{10} \mathrm{GC}\right.$ in $50 \mu \mathrm{l}$ PBS $)$ or mRNA $(20 \mu \mathrm{g})$ were administered via intratracheal highpressure spraying (PennCentury), as described previously (23). Recombinant IL-17A and IL-23 (200 ng in $40 \mu \mathrm{l} \mathrm{PBS}$; BioLegend) were administered intranasally ( $20 \mu \mathrm{l} /$ nostril) on days 26 and 28 . For IL-10 depletion, the antimouse IL-10 mAb, JES5-2A5 (rat IgG1; eBioscience), was delivered on days 25 and 27 . IL-17A $/ /$ mice on a BALB/c background were obtained from Johannes Gutenberg University Mainz.

\section{Airway resistance}

Airway resistance in response to MCh was determined using the ex vivo model of the isolated, perfused, and ventilated lung (IPL) (33, 34). Briefly, mouse lungs placed in a thorax chamber were mechanically ventilated via a tracheal cannula and perfused via the pulmonary artery at a constant flow rate $(1 \mathrm{ml} / \mathrm{min})$ in a nonrecirculating manner with a $4 \%$ hydroxyethyl starch containing perfusion buffer. Lungs were ventilated with 90 breaths per minute by negative pressure ventilation between $-2.8 \mathrm{~cm} \mathrm{H}_{2} \mathrm{O}$ (endexpiratory) and $-8.5 \mathrm{~cm} \mathrm{H}_{2} \mathrm{O}$ (end-inspiratory); hyperinflation $(-25 \mathrm{~cm}$ $\mathrm{H}_{2} \mathrm{O}$ ) was performed every 5 minutes to prevent atelectases. Lung function parameters were analyzed automatically by HSE-HA Pulmodyn W Software (Harvard Apparatus).

After a 20-minute equilibration period, the lungs were perfused with increasing concentrations of $\mathrm{MCh}(0.1 \mu \mathrm{M}, 1 \mu \mathrm{M}, 10 \mu \mathrm{M}$, and $100 \mu \mathrm{M})$ (acetyl- $\beta$-methylcholine chloride; Sigma-Aldrich) for 10 minutes each, separated by a 20 -minute washout period with buffer. For graphical and statistical analysis, the mean resistance values were calculated from the last 30 seconds of each 10-minute MCh exposure.

\section{Endpoints}

$B A L$. Lung lavage was performed through the trachea with $1 \mathrm{ml}$ PBS. Total BAL cells were counted using trypan blue dye exclusion, and differential cell counts were performed on cytospin preparations. Cytokines in BAL supernatant were assayed using ELISAs (eBioscience) for IL-4, IL-5, IL-13, IL-17A, IL-23, and IL-10, according to the manufacturer's protocol. Markers of airway remodeling, MMP-9 (R\&D Systems), TGF $\beta$ (eBioscience) neutrophil elastase, and VEGF (Biozol), were analyzed by ELISA in BAL.

Serum. OVA-specific (MD Biosciences) and total IgE (eBioscience) levels were assayed by ELISA.

Lung single-cell suspension. Harvested lungs were digested at $37^{\circ} \mathrm{C}$ for 1 hour on a rotating shaker in $1 \mathrm{mg} / \mathrm{ml}$ collagenase type I (Life Technologies), $1 \%$ (500 U) DNase (EPICENTRE Biotechnologies) solution. Digested lung was passed through a $40-\mu \mathrm{m}$ nylon cell strainer and erythrocytes were lysed using ACK Lysing Buffer (Life Technologies).

Flow cytometry. Anti-CD3, anti-CD4, anti-CD25, anti-Foxp3, antiIL-17A, anti-CD11b, anti-Ly6G, anti-GR1, anti-F4/80 (eBioscience),
anti-proSPC (Millipore), and anti-FLAG M2 (Sigma-Aldrich) were used to stain lung cells. For intracellular staining, cells were stimulated for 4 hours with cell stimulation and protein transport inhibitor cocktails (eBioscience) according to the manufacturer's instructions. After staining for extracellular markers, cells were fixed, permeabilized, and stained with intracellular antibodies.

Western blot. Protein harvested from lung was run on an SDS-PAGE gel, transferred to a PVDF membrane, and stained with anti-FLAG M2-HRP antibody (1:500; Sigma-Aldrich) according to the manufacturer's instructions.

Histopathology. Paraffin tissue sections stained with either H\&E or PAS were examined using a Zeiss Axio Imager.M2 with the AxioCam MRc camera, where peribronchial inflammation was scored on a scale of 0 to 4 , and PAS-positive goblet cells per $1 \mathrm{~mm}$ of basement membrane were quantified.

Real-time RT-PCR. Total RNA was obtained from lung homogenate using an RNeasy Mini Kit (QIAGEN) and reverse-transcribed into cDNA using a $20-\mu 1$ reaction mixture (Bio-Rad). RT-PCR analyses for Foxp3, Gata3, and Roryt expression were performed on a ViiA 7 cycler (Life Technologies) using QuantiTect Primers (QIAGEN), with $\beta$-actin as a normalizing control.

\section{Statistics}

Relative differences in mRNA expression between groups were analyzed by pairwise fixed reallocation randomization tests using REST 2009 software (35). All other calculations were performed using GraphPad Prism 5.0 software. Statistical significance $(P \leq 0.05)$ was determined using the Mann-Whitney $U$ test, or by a 1-way ANOVA followed by Tukey's multiple comparison test for airway resistance data.

\section{Study approval}

Animal studies were conducted following protocols approved by the Regierungspräsidium Tübingen, Tübingen, Germany.

\section{Acknowledgments}

This work was supported by funding from the Deutsche Forschungsgemeinschaft (KO 4258/2-1 to M.S.D. Kormann and L.E. Mays; Emmy Noether Programme HA 5274/3-1 to D. Hartl); the European Respiratory Society (ERS) Maurizio Vignola Award (to M.S.D. Kormann); the Interfaculty Center for Pharmacogenomics and Drug Research (ICEPHA Twinning grants 12-0-0 and 22-0-0 to S. Beer-Hammer, S. Ammon-Treiber, and M. Schwab); and the Robert Bosch Foundation, Stuttgart (to M. Schwab). L.E. Mays received an ERS/Marie Curie Joint Postdoctoral Research Fellowship (MC 1623-2010), supported by the ERS and the European Community's Seventh Framework Programme FP7/20072013-Marie Curie Actions under grant agreement RESPIRE, PCOFUND-GA-2008-229571. The authors thank Azita Mahiny, Melanie Carevic, Martina Bakele, and Iris Schäfer for their excellent technical support. Special thanks to Y. Iwakura from the Research Institute for Biomedical Sciences at Tokyo University of Science for access to the IL-17A-/- mice used in this study, and to Penn Vector Core for access to the plasmids used in AAV vector production.

Received for publication June 14, 2012, and accepted in revised form December 11, 2012.

Address correspondence to: Michael S.D. Kormann, Department of Pediatrics I, Translational Genomics and Gene Therapy, University of Tübingen, Wilhelmstrasse 31, D-72074 Tübingen, Germany. Phone: 49.07071.2984305; Fax: 49.07071.2984717; E-mail: michael.kormann@med.uni-tuebingen.de. 
1. Elias JA, Lee CG, Zheng T, Ma B, Homer RJ, Zhu $Z$. New insights into the pathogenesis of asthma. J Clin Invest. 2003;111(3):291-297.

2. Locksley RM. Asthma and allergic inflammation. Cell. 2010;140(6):777-783.

3. Hellings PW, et al. Interleukin-17 orchestrates the granulocyte influx into airways after allergen inhalation in a mouse model of allergic asthma. $A m J$ Respir Cell Mol Biol. 2003;28(1):42-50.

4. Wilson RH, Whitehead GS, Nakano H, Free ME, Kolls JK, Cook DN. Allergic sensitization through the airway primes Th17-dependent neutrophilia and airway hyperresponsiveness. Am J Respir Crit Care Med. 2009;180(8):720-730.

5. Molet S, et al. IL-17 is increased in asthmatic airways and induces human bronchial fibroblasts to produce cytokines. J Allergy Clin Immunol. 2001;108(3):430-438

6. Kudo M, et al. IL-17A produced by alphabeta T cells drives airway hyper-responsiveness in mice and enhances mouse and human airway smooth muscle contraction. Nat Med. 2012;18(4):547-554.

7. Wakashin $\mathrm{H}$, et al. IL-23 and Th17 cells enhance Th2-cell-mediated eosinophilic airway inflammation in mice. Am J Respir Crit Care Med. 2008; 178(10):1023-1032.

8. Moreira AP, et al. The protective role of TLR6 in a mouse model of asthma is mediated by IL-23 and IL-17A. J Clin Invest. 2011;121(11):4420-4432.

9. Schnyder-Candrian S, et al. Interleukin-17 is a negative regulator of established allergic asthma. J Exp Med. 2006;203(12):2715-2725.

10. Sakaguchi S, Miyara M, Costantino CM, Hafler DA. Foxp3+ regulatory $\mathrm{T}$ cells in the human immune system. Nat Rev Immunol. 2010;10(7):490-500.

11. Robinson DS. Regulatory T cells and asthma. Clin Exp Allergy. 2009;39(9):1314-1323.

12. Hartl D, et al. Quantitative and functional impairment of pulmonary CD4+CD25hi regulatory $\mathrm{T}$ cells in pediatric asthma. J Allergy Clin Immunol. 2007;119(5):1258-1266.

13. Karagiannidis C, et al. Glucocorticoids upregulate Foxp3 expression and regulatory $\mathrm{T}$ cells in asthma. J Allergy Clin Immunol. 2004;114(6):1425-1433.
14. Kearley J, Barker JE, Robinson DS, Lloyd CM. Resolution of airway inflammation and hyperreactivity after in vivo transfer of CD4+CD25+ regulatory $\mathrm{T}$ cells is interleukin 10 dependent. J Exp Med. 2005;202(11):1539-1547.

15. Lewkowich IP, et al. CD4+CD25+ T cells protect against experimentally induced asthma and alter pulmonary dendritic cell phenotype and function. J Exp Med. 2005;202(11):1549-1561.

16. Zuany-Amorim C, et al. Suppression of airway eosinophilia by killed mycobacterium vaccae-induced allergen-specific regulatory T-cells. Nat Med. 2002;8(6):625-629.

17. Arnold IC, et al. Helicobacter pylori infection prevents allergic asthma in mouse models through the induction of regulatory $\mathrm{T}$ cells. J Clin Invest. 2011;121(8):3088-3093.

18. Doganci A, et al. The IL-6R alpha chain controls lung CD4+CD25+ Treg development and function during allergic airway inflammation in vivo. $J$ Clin Invest. 2005;115(2):313-325.

19. Hammad H, et al. Activation of the D prostanoid 1 receptor suppresses asthma by modulation of lung dendritic cell function and induction of regulatory T cells. J Exp Med. 2007;204(2):357-367.

20. Schreiber TH, et al. Therapeutic Treg expansion in mice by TNFRSF 25 prevents allergic lung inflammation. J Clin Invest. 2010;120(10):3629-3640.

21. Curiel TJ, et al. Specific recruitment of regulatory $\mathrm{T}$ cells in ovarian carcinoma fosters immune privilege and predicts reduced survival. Nat Med. 2004;10(9):942-949.

22. Belkaid Y, Piccirillo CA, Mendez S, Shevach EM, Sacks DL. CD4+CD25+ regulatory T cells control Leishmania major persistence and immunity. Nature. 2002;420(6915):502-507.

23. Kormann MS, et al. Expression of therapeutic proteins after delivery of chemically modified mRNA in mice. Nat Biotechnol. 2011;29(2):154-157.

24. Limberis MP, Vandenberghe LH, Zhang L, Pickles RJ, Wilson JM. Transduction efficiencies of novel AAV vectors in mouse airway epithelium in vivo and human ciliated airway epithelium in vitro. $\mathrm{Mol}$ Ther. 2009;17(2):294-301.
25. Hacein-Bey-Abina S, et al. LMO2-associated clonal $T$ cell proliferation in two patients after gene therapy for SCID-X1. Science. 2003;302(5644):415-419.

26. Zhou L, et al. TGF-beta-induced Foxp3 inhibits $\mathrm{T}(\mathrm{H}) 17$ cell differentiation by antagonizing RORgammat function. Nature. 2008; 453(7192):236-240

27. Nakae S, et al. Antigen-specific T cell sensitization is impaired in IL-17-deficient mice, causing suppression of allergic cellular and humoral responses. Immunity. 2002;17(3):375-387.

28. Huber S, et al. Th17 cells express interleukin-10 receptor and are controlled by Foxp3 and Foxp3+ regulatory $\mathrm{CD} 4+\mathrm{T}$ cells in an interleukin-10-dependent manner. Immunity. 2011;34(4):554-565.

29. Massoud $\mathrm{AH}$, et al. Intravenous immunoglobulin attenuates airway inflammation through induction of forkhead box protein 3-positive regulatory $\mathrm{T}$ cells. J Allergy Clin Immunol. 2012;129(6):1656-1665.

30. Xu W, et al. Adoptive transfer of induced-Treg cells effectively attenuates murine airway allergic inflammation. PLoS One. 2012;7(7):e40314.

31. Zhao J, Lloyd CM, Noble A. Th17 responses in chronic allergic airway inflammation abrogate regulatory $\mathrm{T}$-cell-mediated tolerance and contribute to airway remodeling [published online ahead of print August 15, 2012]. Mucosal Immunol. doi:10.1038/mi.2012.76.

32. McGeachy MJ, et al. TGF-beta and IL- 6 drive the production of IL-17 and IL-10 by T cells and restrain $\mathrm{T}(\mathrm{H})-17$ cell-mediated pathology. Nat Immunol. 2007;8(12):1390-1397.

33. Held HD, Martin C, Uhlig S. Characterization of airway and vascular responses in murine lungs. $\mathrm{BrJ}$ Pharmacol. 1999;126(5):1191-1199.

34. Dong $M$, et al. Pulmonary delivery and tissue distribution of aerosolized antisense 2 -O-Methyl RNA containing nanoplexes in the isolated perfused and ventilated rat lung. Eur J Pharm Biopharm. 2012;81(3):478-485.

35. Pfaffl MW, Horgan GW, Dempfle L. Relative expression software tool (REST) for group-wise comparison and statistical analysis of relative expression results in real-time PCR. Nucleic Acids Res. 2002;30(9):e36 